\title{
A Brief Overview of 5G Research Activities
}

\author{
Pekka Pirinen \\ Centre for Wireless Communications \\ P.O. Box 4500, FI-90014 University of Oulu, Finland \\ Email: pekka.pirinen@ee.oulu.fi
}

\begin{abstract}
This paper summarizes the main initiatives toward 5G wireless communication networks. Emphasis is paid on the program and project activities as well as on the recent literature. A closer look to a wide range of European Union 5G related projects is conducted. Literature review is restricted to recent thematic IEEE Communications Magazine 5G issues and relevant white papers from different sources. The aim is to shed some light on what 5G is about: what are the building blocks of core 5G system concept, what are the main challenges and how to tackle them. The studied references indicate that in addition to capacity boosting technologies 5G needs to offer, e.g., low latency, ultra-reliable communications, and massive connectivity. Thus, the most demanding part in $5 \mathrm{G}$ development will be the design of flexible enough system concept platform that allows successful integration and management of various distinct technologies optimized for diverse use cases.
\end{abstract}

\section{INTRODUCTION}

Increasing Internet data traffic has driven the capacity demands for currently deployed $3 \mathrm{G}$ and $4 \mathrm{G}$ wireless technologies. Now, intensive research toward 5 th generation wireless communication networks is progressing in many fronts. $5 \mathrm{G}$ technology is expected to be in use around 2020. This paper scratches the surface on various $5 \mathrm{G}$ activities by reviewing a wide range of European research projects, recent literature and $5 \mathrm{G}$ white papers from key players in the wireless technology. The intention is to help understanding what $5 \mathrm{G}$ is about and how different $5 \mathrm{G}$ initiatives aim at getting there.

There is no unique definition (yet) for 5G [1], [2]. However, a general consensus is building around the idea that $5 \mathrm{G}$ is merely integration of several techniques, scenarios and use cases rather than the invention of a new single radio access technology. As technical requirements over currently existing technologies (4G) [3] lists the following:

- 1000 times higher mobile data volume per area,

- 10 to 100 times higher typical user data rate,

- 10 to 100 times higher number of connected devices,

- 10 times longer battery life for low power devices,

- 5 times reduced end-to-end latency.

The rest of the paper is organized as follows. Section II picks up the brief essentials of various ongoing $5 \mathrm{G}$ related European projects. Section III reviews IEEE Wireless Communications Magazine thematic issue on 5G wireless communication systems: prospects and challenges, Part 1 in February 2014 [4]-[13] and Part 2 in May 2014 [14]-[22] and finally selected $5 \mathrm{G}$ white papers [23]-[29]. Then, Section IV draws concluding remarks followed by the biography.

\section{EURopeAn 5G PROJECTS}

This section overviews mainly European Union 7th Framework Programme (FP7) Future Networks Cluster Radio Access and Spectrum projects [30] managing a large part of European $5 \mathrm{G}$ research activities. New research programs and actions are coordinated by Horizon 2020 [31] and 5G Infrastructure Public Private Partnership (5GPPP) [32].

Mobile and wireless communications Enablers for the Twenty-twenty Information Society (METIS) is the largest FP7 5G project (29 partners) [33], [14] whose objective is to create foundation for $5 \mathrm{G}$ systems and act as a consensus builder toward standardization. METIS has identified some test cases and scenarios to illustrate and address the key challenges $5 \mathrm{G}$ will face. The scenarios are listed as:

1) 'Amazingly fast',

2) 'Great service in a crowd',

3) 'Best experience follows you',

4) 'Super real-time and reliable connections', and

5) 'Ubiquitous things communicating'.

The first one focuses on providing very high data rates with very low latency (instantaneous connectivity). The second scenario should ensure reasonable user experiences in densely populated areas like shopping malls, stadiums and rock concerts. The third scenario has emphasis on user mobility, e.g., vehicular communication, again with high service experience. The fourth scenario accounts for new use cases/applications that have stringent requirements for reliability and latency. The last scenario deals with the efficient management of a very large number devices (e.g., machines, sensors).

METIS has also identified so-called horizontal topics (HTs) to be integrated with technology components to form the overall system concept. These are:

1) direct device-to-device (D2D) communication,

2) massive machine communication $(M M C)$,

3) moving networks ( $M N s$ ),

4) ultra-dense networks (UDNs), and

5) ultra-reliable communication (URC).

Finally, there is an architectural framework to combine various centralized and decentralized approaches into a consistent concept.

According to [34] METIS has selected technology components "Direct network controlled device to device communication with interference cancellation" and "FBMC/OQAM new waveform" for test-beds as they are expected to have high technology impact and relevance for the $5 \mathrm{G}$ system concept. 
5th Generation Non-Orthogonal Waveforms for Asynchronous Signalling (5GNOW) project [35], [8] proposes a scalable and efficient air interface that gives up on strict orthogonality and synchronism principles followed in former generation networks. Universal filtered multi-carrier (UFMC), filter bank multi-carrier (FBMC) and generalized frequencydivision multiplexing (GFDM) are few examples of promising waveforms that can outperform orthogonal frequency division multiplexing (OFDM) in 5G context. In addition to multicarrier waveform design itself, 5GNOW addresses such aspects as a unified frame structure, filtering, sparse signal processing, robustness, and very low latency transmissions.

Enhanced Multicarrier Technology for Professional Adhoc and Cell-Based Communications (EMPhAtiC) project [36] developes highly flexible and efficient filter-bank processing structure and channel estimation, equalization and synchronization functionalities needed therein. Furthermore, feasibility of filterbank based multicarrier schemes in some specific environments (relays, multihop, cooperative), where synchronization is hard to maintain, will be evaluated.

Energy Efficient E-band Transceiver for Backhaul of the Future Networks (E3NETWORK) project's [37] transceiver design aims at high spectral and energy efficiency by using modern digital multi-level modulations and highly integrated circuits (advanced SiGe BiCMOS technology) in the RF analogue front-end.

PHYsical LAyer Wireless Security (PHYLAWS) project [38] aims to enhance radio interface privacy in wireless networks via physical layer security and secrecy coding approaches. Trustworthy radio waveforms and access protocols are designed and tested in realistic experimental WiFi setups and LTE simulation cases.

Full-Duplex Radios for Local Access (DUPLO) project [39] builds upon radio transceiver technology where the same carrier frequency can be used simultaneously for transmission and reception. This new transmission paradigm can significantly boost link capacity and provide new means for flexible spectrum use and networking.

Connectivity management for eneRgy Optimised Wireless Dense networks (CROWD) project [40] aims at very dense heterogeneous access networks integrated with wireless/wired backhaul capabilities. The goals are to provide density-proportional capacity where needed, optimize MAC mechanisms for such environments, enable traffic-proportional energy consumption, and ensure user quality of experience via smart connectivity management designs.

Dense Cooperative Wireless Cloud Network (DIWINE) project [41] utilizes the paradigm of "virtual relay based selfcontained wireless cloud', which has a simple and unambiguous interface to terminals, in solving the problem of wireless communications in densely interfering ad hoc networks.

Interworking and JOINt Design of an Open Access and Backhaul Network Architecture for Small Cells based on Cloud Networks (iJOIN) project [42] will utilize centralized open IT platform based radio access network (RAN) concept that has a cloud infrastructure. Access and backhaul structure is jointly designed and optimized.

Distributed computing, storage, and resource allocation over cooperative femtocells (TROPIC) project [43] seeks answers to the following questions: What kind of communication/computation technologies are needed to converge pervasive femto-network infrastructure and cloud computing? How much gain in spectral/energy/service efficiency can be achieved via the proposed femto-cloud methods?

Wireless technologies for isolated rural communities in developing countries based on cellular $3 G$ femtocell deployments (TUCAN3G) project [44] takes advantage of new wireless access ( $3 \mathrm{G} \rightarrow 4 \mathrm{G}$ femtocells) and heterogeneous backhauling technologies (long distance WiFi, WiMAX, VSAT) in outdoor scenarios to establish economically sustainable and technologically feasible solution for rural environments.

Beyond 2020 Heterogeneous Wireless Networks with Millimeter-Wave Small Cell Access and Backhauling (MiWaveS) [45] is an industry-driven large-scale integrating project. It investigates and demonstrates key enabling technologies and functionalities supporting the integration of millimeter-wave small-cells in future heterogeneous networks. The project's interest is particularly at the level of networking functions and algorithms, and integrated radio and antenna technologies.

Advanced Dynamic spectrum 5 G mobile networks Employing Lisensed shared access (ADEL) project [46] has focus on investigating the following research problems within the lisensed shared access (LSA) paradigm: 1) the dynamic and optimized allocation of spectral and power resources at time scales from seconds to even milliseconds, 2) quality of service guarantees to the users of all participating spectrum-sharing networks, and 3) overall energy expenditure minimization of LSA networks.

Spectrum OverLay through aggregation of heterogeneous DispERsed bands (SOLDER) project's [47] main goal is to efficiently aggregate of non-continuous dispersed spectrum bands licensed to heterogeneous networks (HetNets) and heterogeneous Radio Access Technologies (h-RATs) and thereby come up with a new spectrum overlay technology.

Cognitive Radio Standardization initiative (CRS-i) coordination action [48] coordinates and supports current and future FP7 projects. Their cognitive radio and dynamic spectrum access results are exploited via concentrated approach and prepared toward standardization.

COgnitive RAdio for SATellite Communications (CoRaSat) project [49] studies dynamic spectrum sharing by investigating, developing, and demonstrating relevant cognitive radio techniques to satellite networks. The intention is to demonstrate that benefits and new business opportunities of flexible spectrum usage will outweigh their potential drawbacks.

Self-Management for Unified Heterogeneous Radio Access Networks (SEMAFOUR) project's [50] objectives are to develop multiple radio access technology (multi-RAT) and multi-layer self-organizing networks (SON) functions (incorporating closed control loop for the configuration, optimiza- 
tion and failure recovery) and to design an integrated SON management system.

Massive MIMO for Efficient Transmission (MAMMOET) project [51] aims to bring massive MIMO from highly promising theoretical concept to practically implementable level by using innovative low-cost yet efficient and flexible hardware.

High capacity network Architecture with Remote radio heads \& Parasitic antenna arrays (HARP) project [52] plans to realize distributed multi-antenna wireless access by combining radio remote heads (RRHs) technology with electronically steerable passive array radiators (ESPARs) technology. This would spread radio-over-fibre connections geographically over wide area distributed access via radio-over-fibre and provide with a single active RF chain multi-antenna-like functionality.

Mobile Cloud Networking (MCN) project [53] creates cloud-based elastic mobile networks on-demand. Specific interest is on evolved packet systems and their lifecycle management.

Evolving mobile internet with innovative terminal-toterminal offloading technologies (MOTO) project [54] intends to tackle $5 \mathrm{G}$ challenges by exploiting diverse set of offloading schemes, e.g., cellular to Wi-Fi, and by opportunistically utilizing D2D connections. Network controlled IP traffic offloading is also incorporated.

European Cooperation in Science and Technology (COST) action on Cooperative Radio Communications for Green Smart Environments (COST IC1004) [55], as the name suggests, has the scientific scope on energy efficient radio communication systems and networks and smart environments.

Network of Excellence in Wireless Communications (NEWCOM\#) project [56] concentrates on long-term and interdisciplinary research. Examples of the research targets are exploring the ultimate limits of communication networks, energy and spectral efficient communications and networking as well as opportunistic and cooperative communications. Moreover, NEWCOM\# encourages cooperation between academy and industry and educates new researchers, e.g., by organizing seasonal schools.

SOftware-Defined Access using Low-Energy Subsystems (SODALES) project [57] aims to develop a novel wireless access interconnection service that has a low-cost $10 \mathrm{Gbps}$ fixed access target and offers transparent transport services for fixed and mobile subscribers. The convergent network architecture includes advanced control and management plane with open-access and multi-operator functionalities.

Links-on-the-fly Technology for Robust, Efficient and Smart Communication in Unpredictable Environments (RESCUE) project [58] sees future heterogeneous and dense networks to form an appropriate basis for lossy communication links based network concept. Public safety and vehicle-tovehicle applications are in the forefront in RESCUE.

Aerial Base Stations with Opportunistic Links for Unexpected \& Temporary Events (ABSOLUTE) project [59] focuses on future public safety communication systems. The proposed heterogeneous network architecture is based on the following elements: 1) low altitude aerial LTE-A base stations
(AeNodeB), 2) portable land mobile LTE-A base stations (TeNodeB), and 3) advanced multimode LTE-A professional terminals.

Low EMF Exposure Future Networks (LEXNET) project [60], [61] has a very concrete target to develop effective mechanisms to reduce at least $50 \%$ of the public electromagnetic field (EMF) exposure without jeopardizing the quality of service.

\section{LITERATURE REVIEW ON 5G}

A vast body of publications is relevant for $5 \mathrm{G}$ and more articles come out every month. Therefore, the literature selected in this section is restricted to very recent popular magazine level articles and selected white papers. More specifically, IEEE Communications Magazine has issued a two-part feature topic on 5G in February and May 2014, respectively, and the papers therein are briefly summarized here.

\section{A. Overview of IEEE Communications Magazine Feb. 2014} $5 G$ Section

Paper [4] challenges us to rethink relationship between energy and spectral efficiency (EE vs. SE). Co-design of these should be important part of $5 \mathrm{G}$ research. The ideal future system should have EE improvement for each SE point, larger win-win and smaller EE-SE trade-off region and smaller slope in EE-SE trade-off region. No more cells is another statement that suggests $5 \mathrm{G}$ to shift from cell-centric thinking towards soft user and C-RAN centric designs. The third point is to rethink signaling and control mechanisms for diverse traffic types. As the fourth aspect [4] introduces the concept of invisible base stations. It covers the deployment of massive MIMO in the form of irregular antenna arrays where antenna elements can be embedded into the environment (thus making base stations virtually invisible). Finally, full duplex radio is proposed as one useful technology component for $5 \mathrm{G}$.

Similarly to the previous article, Boccardi et al. in [5], list five disruptive viewpoints toward 5G. Conventional base station based cellular structures (up/downlink, control/data channels) are expected to give way to more agile device-centric architectures where diverse nature of traffic and network nodes can be handled better. Additional broad bandwidths are available in millimeter waves and should be taken into use. Massive MIMO has potential for $5 \mathrm{G}$ as it is scalable technology at node level and enables new deployments and architectures. Devices are getting more intelligent and that should be reflected both at node and higher architectural level. As an example, D2D connectivity and mobile device caching have implications on $5 \mathrm{G}$ system design. An integral part of 5G should also be natural support for machine-to-machine (M2M) communication where the number of connected devices can be extremely large and high reliability and low latency are required.

Network densification is the main focus area of [6]. The two dimensions in this trend are over space and over frequency. Spatial domain is mostly covered by dense deployment of small cells whereas frequency domain densification comprises 
of aggregating larger chunks of radio spectrum from diverse bands into effective use. In parallel, high-capacity low-latency backhaul need to be developed to guarantee enhanced user experience.

Reference [7] makes the following key observations: 1) macrocellular capacity increase is likely to reach its limits, 2) measures for mobile performance require updating, and 3) the variety of both the radio access technologies and the devices is increasing. 5G era needs co-optimization of networks, devices, and applications to achieve required improvements in service performance and efficiency.

$5 \mathrm{GNOW}$ project's vision on $5 \mathrm{G}$ waveform design is reflected in [8]. The idea is to loosen the synchronism and orthogonality requirement by design and allow a controllable amount of waveform crosstalk. The resulting multi-carrier waveforms have some competitive edge over well-established OFDM technology.

Lower end of the frequency spectrum has already been reserved to a large extent for various legacy systems. Therefore, broad contiguous bandwidths are only available at high center frequencies, e.g., millimeter waves. Reference [9] delves into millimeter-wave beamforming and its feasibility as a candidate technology for $5 \mathrm{G}$. In addition to the theoretical prospects, prototyping status is reviewed so that practical aspects of millimeter-wave communications become addressed as well.

Full duplex (FD) technology is one potential building block to be considered for 5G. Paper [10] concentrates on this technology and especially in self-interference mitigation that must be effective in FD systems to make them practical.

Reference [11] differentiates outdoor and indoor scenarios in 5G cellular architecture design to avoid high wallpenetration losses. Distributed antenna system (DAS) and massive MIMO technologies help in this. Indoor coverage can be provided via such short-range wireless technologies as WiFi, femtocells, visible light communication (VLC), and $\mathrm{mm}$-waves whereas outdoor users are served by heterogeneous architecture including large MIMO networks, mobile femtocells and cognitive radio networks.

Caching popular content in intermediate servers decreases redundant traffic in core network and thus alleviates network congestion problems. Current and foreseeable caching techniques for $5 \mathrm{G}$ are discussed in [12].

Fettweis and Alamouti [13] acknowledge that cellular telephony first had huge impact on voice services and later on data delivery. However, regarding Internet there is still much to be exploited by $5 \mathrm{G}$ technologies. As a result a user-centric future tactile Internet will utilize technological advancements in content delivery, control (response times) and monitoring (endurance).

\section{B. Overview of IEEE Communications Magazine May 2014 $5 G$ Section}

The second thematic 5G issue is opened by the METIS project vision about methodology, requirements and scenarios for future wireless access networks [14]. METIS scenarios and horizontal drivers were already described in Section II.
The next article [15] proposes a change in evolution paths toward 5G. The past generations have been dominated by macrocell development but coordinated macro/local coexistence would be more fruitful direction in the future.

Improvements in the spectral efficiency and coverage of the cell edge users are the main targets in [16]. As a tool to achieve the goal is a smart combination of small cell deployment, joint transmission coordinated multipoint (JT CoMP), and massive MIMO techniques with affordable complexity.

Advanced interference management is seen in [17] as an important initial technology driver toward $5 \mathrm{G}$. This paper investigates interference joint detection/decoding in user equipment and joint scheduling as network-side interference management scheme.

Paper [18] promotes cell densification via hyper-dense small cell deployment. The scenarios are divided into residential, enterprise, and hotspot deployments that have their own service requirements. Synchronization, graph-based carrier selection and intercell power control are also important aspects in cooperative distributed small cell optimization.

Cloud technologies are rapidly emerging in modern Internet usage and inevitably will have a role in $5 \mathrm{G}$ radio access networks as well. Reference [19] presents a radio access network (RAN) as a service (RANaaS) concept as a flexible centralized processing platform for $5 \mathrm{G}$.

Public-private spectrum sharing is seen in [20] to be a realistic way of providing high quality of experience (QoE) required by $5 \mathrm{G}$.

Various D2D communication categories and their specific challenges are discussed in [21]. Communication modes are divided into operator and device controlled schemes. In addition to technical operation the pricing of D2D services is evaluated.

The final article in this series [22] is about designing a framework for cooperative green heterogeneous networks to find balance between spectral and energy efficiency and quality of service.

\section{C. $5 G$ White Papers}

4G Americas has very recently published a 44 page summary of global 5G initiatives [23] that overviews regional 5G activities in Europe, Asia and America. In addition to research projects, 5G related actions in standardization bodies (e.g., 3GPP, ITU, IEEE), associations (e.g., TIA), alliances (e.g., NGMN and WWRF) and industry are listed.

Nokia Solutions and Networks white paper [24] shares the view that $5 \mathrm{G}$ is unlikely to be just a single new radio access technology (RAT) but more like a combination of existing air interface technologies both in licensed and unlicensed bands with some novel technologies optimized for specific use cases and scenarios (e.g., ultra-dense deployments). Furthermore, more spectrum and small cell base stations are needed (network densification) and network performance needs enhancements (e.g., virtual zero latency) to make $5 \mathrm{G}$ reality.

Huawei has defined so-called "5G HyperService Cube" [25] to show 3D-illustration of how different types of ser- 


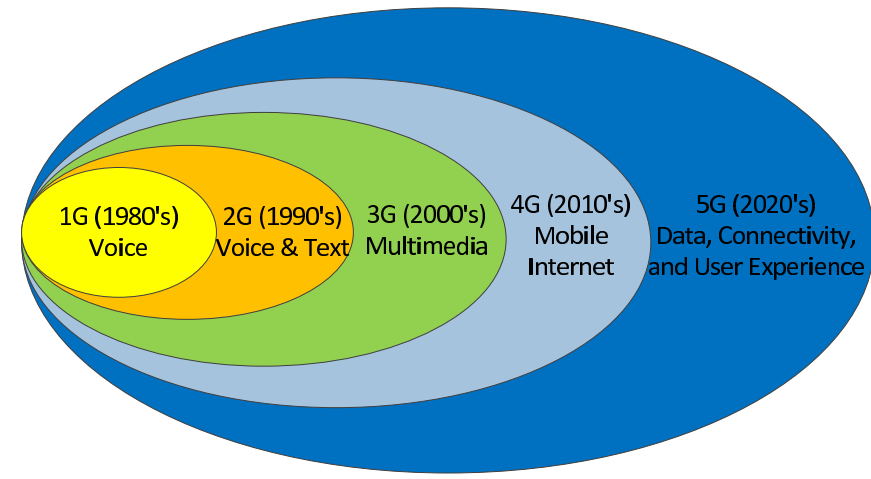

Fig. 1. Development of service types over wireless mobile generations.

vices fit inside it. Dimensions in the cube are 1) throughput $\left.\left(\mathrm{kbps} / \mathrm{km}^{2}\right), 2\right)$ delay $(\mathrm{ms})$, and 3) number of links (per $\mathrm{km}^{2}$ ). As an example multi-user ultra high definition telepresence and smart sensors lie in the opposite corners of the cube as the former requires extremely high throughput and low number of connections and low latency whereas the latter service can tolerate large delay with low throughput but requires a large amount of links. Main design objectives for $5 \mathrm{G}$ are: 1) realization of massive capacity and connectivity, 2) support to very diverse set of services, applications, users and requirements, and 3 ) efficient and flexible utilization of all available non-contiguous spectrum resources.

Ericsson [26] reckons the following properties to be integral part of 5G: very high mobile broadband service level everywhere, ultra-high traffic capacity and data rates, huge number of low-power machine-type communication devices, proximal communication, ultra-reliable communication, energy efficiency and sustainability, and new spectrum assignments.

Datang white paper [27] sees evolution, convergence and innovation to form the technology routes toward 5G. Fig. 1 shows how supported services evolve from $1 \mathrm{G}$ speech delivery via $3 \mathrm{G}$ multimedia up to versatility of $5 \mathrm{G}$ networks.

ZTE [28] sees user experience being in focus of $5 \mathrm{G}$ research instead of only increasing network capacity. From the technical point of view the main challenges are: 1) massive traffic volumes, 2) gradual transfer to an intelligent cloud architecture, 3) thorough convergence of networks and services, and 4) convert networks to have much more configurable capabilities.

NTT DOCOMO [29] highlights the importance of lower and higher frequency bands' effective integration in order to achieve the intended 5G system capacity (1000-fold) and typical data rate (100-fold). This can be supported by phantom cell concept (where control and user planes are separated) and by flexible duplexing. In high frequencies new numerology and waveform design is needed and in lower frequencies nonorthogonal multiple access (NOMA) can be utilized.

\section{CONCLUSION}

This paper gives an overview of different $5 \mathrm{G}$ activities around the world and particularly in Europe. The special attention is paid on key literature, projects and programs focusing on $5 \mathrm{G}$ technology. Although the $5 \mathrm{G}$ concept is still evolving the review reveals emerging common features. Performance enhancements are mainly expected from a combination of network densification (e.g., small cells, D2D), increased spectrum (enhanced carrier aggregation, spectrum sharing, beyond $6 \mathrm{GHz}$ frequencies), and enhanced wireless communication technologies (e.g., massive MIMO, new waveforms, virtual zero latency RATs). Machine-type of communications will have increasing proportion of the network connections and traffic. Combination of moving networks and ultra reliable communications truly calls for novel solutions due to strict technical requirements in challenging propagation conditions. Network virtualization, especially in the form of Cloud RAN development will also have a significant role in $5 \mathrm{G}$. Use cases, scenarios and spectrum allocations altogether have so high variability that utmost agility, scalability and reconfigurability is necessary in the integration of the overall $5 \mathrm{G}$ system concept.

\section{ACKNOWLEDGMENT}

The author would like to thank the Finnish Funding Agency for Technology and Innovation (Tekes), Nokia, Huawei, Broadcom and Elektrobit for $5 \mathrm{Gto} 10 \mathrm{G}$ project funding enabling this work. Special thanks go to Eric Galloix, Kari Pajukoski, Tao Cai and anonymous reviewers for constructive comments.

\section{REFERENCES}

[1] 5G wikipedia page. [Online]. Available: http://en.wikipedia.org/wiki/5G

[2] J. G. Andrews, S. Buzzi, W. Choi, S. V. Hanly, A. Lozano, A. C. K. Soong, and J. C. Zhang, "What will 5G be?" IEEE J. Sel. Areas Commun., vol. 32, pp. 1065-1082, Jun. 2014.

[3] P. Popovski, V. Braun, H.-P. Mayer, P. Fertl, Z. Ren, D. GozalvesSerrano, E. Ström, T. Svensson, H. Taoka, P. Agyapong, A. Benjebbour, G. Zimmermann, J. Meinilä, J. Ylitalo, T. Jämsä, P. Kyösti, K. Dimou, M. Fallgren, Y. Selén, B. Timus, H. Tullberg, M. Schellmann, Y. Wu, M. Schubert, D. H. Kang, J. I. Markendahl, C. Beckman, M. Uusitalo, O. Yilmaz, C. Wijting, Z. Li, P. Marsch, K. Pawlak, J. Vihriälä, A. Gouraud, S. Jeux, M. Boldi, G. M. Dell'aera, B. Melis, H. Schotten, P. Spapis, A. Kaloxylos, and K. Chatzikokolakis, "ICT-317669-METIS/D1.1 V1 Scenarios, requirements and KPIs for 5G mobile and wireless system," Tech. Rep., May 2013. [Online]. Available: https://www.metis2020.com/wp-content/uploads/deliverables/ METIS_D1.1_v1.pdf

[4] C.-L. I, C. Rowell, S. Han, Z. Xu, G. Li, and Z. Pan, "Toward green and soft: a $5 \mathrm{G}$ perspective," IEEE Commun. Mag., vol. 52, pp. 66-73, Feb. 2014.

[5] F. Boccardi, R. W. Heath Jr., A. Lozano, T. L. Marzetta, and P. Popovski, "Five disruptive technology directions for 5G," IEEE Commun. Mag., vol. 52, pp. 74-80, Feb. 2014.

[6] N. Bhushan, J. Li, D. Malladi, R. Gilmore, D. Brenner, A. Damnjanovic, R. T. Sukhavasi, C. Patel, and S. Geirhofer, "Network densification: the dominant theme for wireless evolution into 5G," IEEE Commun. Mag., vol. 52, pp. 82-89, Feb. 2014.

[7] B. Bangerter, S. Talwar, R. Arefi, and K. Stewart, "Networks and devices for the 5G era," IEEE Commun. Mag., vol. 52, pp. 90-96, Feb. 2014.

[8] G. Wunder, P. Jung, M. Kasparick, T. Wild, Y. Chen, S. ten Brink, I. Gaspar, N. Michailow, A. Festag, L. Mendes, N. Cassiau, D. Kténas, M. Dryjanski, S. Pietrzyk, B. Eged, P. Vago, and F. Wiedmann, "5GNOW: non-orthogonal, asynchronous waveforms for future mobile applications," IEEE Commun. Mag., vol. 52, pp. 97-105, Feb. 2014.

[9] W. Roh, J.-Y. Seol, J. Park, B. Lee, J. Lee, Y. Kim, J. Cho, K. Cheun, and F. Aryanfar, "Millimeter-wave beamforming as an enabling technology for 5G cellular communications: theoretical feasibility and prototype results," IEEE Commun. Mag., vol. 52, pp. 106-113, Feb. 2014. 
[10] S. Hong, J. Brand, J. I. Choi, M. Jain, J. Mehlman, S. Katti, and P. Levis, "Applications of self-interference cancellation in $5 \mathrm{G}$ and beyond," IEEE Commun. Mag., vol. 52, pp. 114-121, Feb. 2014.

[11] C.-X. Wang, F. Haider, X. Gao, X.-H. You, Y. Yang, D. Yuan, H. M. Aggoune, H. H. S. Fletcher, and E. Hepsaydir, "Cellular architecture and key technologies for $5 \mathrm{G}$ wireless communication networks," IEEE Commun. Mag., vol. 52, pp. 122-130, Feb. 2014.

[12] X. Wang, M. Chen, T. Taleb, A. Ksentini, and V. C. M. Leung, "Cache in the air: exploiting content caching and delivery techniques for $5 \mathrm{G}$ systems," IEEE Commun. Mag., vol. 52, pp. 131-139, Feb. 2014.

[13] G. Fettweiss and S. Alamouti, "5G: personal mobile internet beyond what cellular did to telephony," IEEE Commun. Mag., vol. 52, pp. 140145, Feb. 2014.

[14] A. Osseiran, F. Boccardi, V. Braun, K. Kusume, P. Marsch, M. Maternia, O. Queseth, M. Schellmann, H. Schotten, H. Taoka, H. Tullberg, M. A. Uusitalo, B. Timus, and M. Fallgren, "Scenarios for $5 \mathrm{G}$ mobile and wireless communications: the vision of the METIS project," IEEE Commun. Mag., vol. 52, pp. 26-35, May 2014.

[15] S. Chen and J. Zhao, "The requirements, challenges, and technologies for $5 \mathrm{G}$ of terrestrial mobile telecommunication," IEEE Commun. Mag., vol. 52, pp. 36-43, May 2014.

[16] V. Jungnickel, K. Manolakis, W. Zirwas, B. Panzner, V. Braun, M. Lossow, M. Sternad, R. Apelfröjd, and T. Svensson, "The role of small cells, coordinated multipoint, and massive MIMO in 5G," IEEE Commun. Mag., vol. 52, pp. 44-51, May 2014.

[17] W. Nam, D. Bai, J. Lee, and I. Kang, "Advanced interference management for $5 \mathrm{G}$ cellular networks," IEEE Commun. Mag., vol. 52, pp. 52-60, May 2014

[18] J. Xu, J. Wang, Y. Zhu, Y. Yang, X. Zheng, S. Wang, L. Liu, K. Horneman, and Y. Teng, "Cooperative distributed optimization for the hyperdense small cell deployment," IEEE Commun. Mag., vol. 52, pp. 61-67, May 2014.

[19] P. Rost, C. J. Bernandos, A. De Domenico, M. Di Girolamo, M. Lalam, A. Maeder, D. Sabella, and D. Wübben, "Cloud technologies for flexible 5G radio access networks," IEEE Commun. Mag., vol. 52, pp. 68-76, May 2014.

[20] J. Mitola III, J. Guerci, J. Reed, Y.-D. Yao, Y. Chen, T. C. Clancy, J. Dwyer, H. Li, H. Man, R. McGwier, and Y. Guo, "Accelerating 5G QoE via public-private spectrum sharing," IEEE Commun. Mag., vol. 52, pp. 77-85, May 2014

[21] M. Nader Tehrani, M. Uysal, and H. Yanikomeroglu, "Device-to-device communication in $5 \mathrm{G}$ cellular networks: challenges, solutions, and future directions," IEEE Commun. Mag., vol. 52, pp. 86-92, May 2014.

[22] R. Q. Hu and Y. Qian, "An energy efficient spectrum efficient wireless heterogeneous network framework for 5G systems," IEEE Commun. Mag., vol. 52, pp. 94-101, May 2014

[23] "4G Americas' summary of global 5G initiatives," White Paper, 4G Americas, Jun. 2014. [Online]. Available: http://www.4gamericas.org/ documents/2014_4GA Summary of Global 5G Initiatives_Final.pdf

[24] "Looking ahead to 5G - building a virtual zero latency gigabit experience," White Paper, Nokia Solutions and Networks, Dec. 2013. [Online]. Available: http://nsn.com/sites/default/files/document/nsn_5g white_paper.pdf

[25] "5G: a technology vision," White Paper, Huawei, Nov. 2013. [Online]. Available: http://www.huawei.com/5gwhitepaper

[26] "5G radio access," White Paper, Ericsson, Jun. 2013. [Online]. Available: http://www.ericsson.com/res/docs/whitepapers/wp-5g.pdf

[27] "Evolution, converge, and innovation - 5G white paper," White Paper, Datang Wireless Mobile Innovation Center, Dec. 2013. [Online]. Available: http://www.datanggroup.cn/upload/accessory/ 201312/2013129194455265372.pdf

[28] "5G - driving the convergence of the physical and digital worlds," White Paper, ZTE, Feb. 2014. [Online]. Available: http://wwwen.zte.com.cn/ en/products/bearer/201402/P02014022140221415329571322.pdf

[29] "5G radio access: requirements, concepts and technologies," White Paper, NTT DOCOMO, Inc., Jul. 2014. [Online]. Available: https://www.nttdocomo.co.jp/english/binary/pdf/corporate/ technology/whitepaper_5g/DOCOMO_5G_White_Paper.pdf

[30] "5G radio network architecture," White Paper, Radio Access and Spectrum FP7 - Future Networks Cluster, Feb. 2014. [Online]. Available: http://www.ict-ras.eu/

[31] Horizon 2020 - The EU Framework Programme for Research and Innovation. [Online]. Available: http://ec.europa.eu/programmes/ horizon2020/
[32] The 5G Infrastructure Public Private Partnership. [Online]. Available: http://5g-ppp.eu/

[33] FP7 Integrating Project METIS (ICT 317669). [Online]. Available: https://www.metis2020.com/

[34] P. Popovski, V. Braun, G. Mange, P. Fertl, D. Gozalves-Serrano, N. Bauer, H. Droste, A. Roos, G. Zimmermann, M. Fallgren, A. Höglung, H. Tullberg, S. Jeux, Ö. Bulacki, J. Eichinger, Z. Li, P. Marsch, K. Pawlak, M. Boldi, and J. F. Monserrat, "ICT317669-METIS/D6.2 V1 Initial report on horizontal topics, first results and 5G system concept," Tech. Rep., Apr. 2014. [Online]. Available: https://www.metis2020.com/wp-content/uploads/deliverables/ METIS_D6.2_v1.pdf

[35] FP7 STReP project 5GNOW (ICT 318555). [Online]. Available: http://www.5gnow.eu/

[36] FP7 STReP project EMPhAtiC (ICT 318362). [Online]. Available: http://www.ict-emphatic.eu/

[37] FP7 STReP project E3NETWORK (ICT 317957). [Online]. Available: http://www.ict-e3network.eu/

[38] FP7 STReP project PHYLAWS (ICT 317562). [Online]. Available: http://www.phylaws-ict.org/

[39] FP7 STReP project DUPLO (ICT 316369). [Online]. Available: http://www.fp7-duplo.eu/

[40] FP7 STReP project CROWD (ICT 318115). [Online]. Available: http://www.ict-crowd.eu/

[41] FP7 STReP project DIWINE (ICT 318177). [Online]. Available: http://diwine-project.eu/

[42] FP7 STReP project iJOIN (ICT 317941). [Online]. Available: http://www.ict-ijoin.eu/

[43] FP7 STReP project TROPIC (ICT 318784). [Online]. Available: http://www.ict-tropic.eu/

[44] FP7 STReP project TUCAN3G (ICT 601102). [Online]. Available: http://www.ict-tucan3g.eu/

[45] FP7 Integrating Project MiWaveS (ICT 619563). [Online]. Available: http://www.miwaves.eu/

[46] FP7 STReP project ADEL (ICT 619647). [Online]. Available: http://www.fp7-adel.eu/

[47] FP7 STReP project SOLDER (ICT 619687). [Online]. Available: http://ict-solder.eu/

[48] FP7 Coordination Action CRS-i (ICT 318563). [Online]. Available: http://www.ict-crsi.eu/

[49] FP7 STReP project CoRaSat (ICT 316779). [Online]. Available: http://www.ict-corasat.eu/

[50] FP7 STReP project SEMAFOUR (ICT 316384). [Online]. Available: http://fp7-semafour.eu/

[51] FP7 STReP project MAMMOET (ICT 619086). [Online]. Available: http://www.mammoet-project.eu/

[52] FP7 STReP project HARP (ICT 318489). [Online]. Available: http://www.fp7-harp.eu/

[53] FP7 Integrating Project MCN (ICT 318109). [Online]. Available: http://www.mobile-cloud-networking.eu/

[54] FP7 STReP project MOTO (ICT 317959). [Online]. Available: http://www.fp7-moto.eu/

[55] COST Action IC1004. [Online]. Available: http://www.ic1004.org/

[56] FP7 NoE project NEWCOM\# (ICT 318306). [Online]. Available: http://www.newcom-project.eu/

[57] FP7 STReP project SODALES (ICT 318600). [Online]. Available: http://www.fp7-sodales.eu/

[58] FP7 STReP project RESCUE (ICT 619555). [Online]. Available: http://www.ict-rescue.eu/

[59] FP7 Integrating Project ABSOLUTE (ICT 318632). [Online]. Available: http://www.absolute-project.eu/

[60] FP7 Integrating Project LEXNET (ICT 318273). [Online]. Available: http://www.lexnet-project.eu/

[61] M. Tesanovic, E. Conil, A. De Domenico, R. Agüero, F. Freudenstein, L. M. Correia, S. Bories, L. Martens, P. M. Wiedemann, and J. Wiart, "The LEXNET project: wireless networks and EMF: paving the way for low-EMF networks of the future," IEEE Veh. Technol. Mag., vol. 9, pp. 20-28, Jun. 2014. 